\title{
Rapid-access gynecological oncology clinic outcomes in North London, UK
}

\section{Jassimran K Bansal 1,* Isabelle G Goldrick',* Ranjit Manchanda ${ }^{2-4}$ Adeola Olaitan ${ }^{5}$}

'Medical School, University College London, ${ }^{2}$ Barts Cancer Institute, Queen Mary University of London, London, ${ }^{3}$ Department of Gynaecological Oncology, Barts Health NHS Trust, Royal London Hospital, London, ${ }^{4}$ Gynaecological Cancer Research Centre, University College London, London, ${ }^{5}$ Department of Gynaecological Oncology, University College London Hospital, London, UK

*These authors contributed equally to this work
Correspondence: Adeola Olaitan Department of Gynaecological Oncology, University College London Hospital, 2nd Floor North, 250 Euston Road, London NWI 2PG, UK

Tel +442034478636

Email adeola.olaitan@uclh.nhs.uk
This article was published in the following Dove Press journal:

Clinical Audit

7 June 2017

Number of times this article has been viewed

Abstract: The North London Gynaecological Cancer Network guidelines, based on Department of Health recommendations, state that women with lesions or symptoms suspicious of gynecological cancer should be referred to the rapid-access clinic (RAC) and see a specialist within 2 weeks. The appropriateness and clinical outcomes of these referrals were evaluated and compared with cancers diagnosed from other sources in the same hospital over the same time period. This was a retrospective review of RAC gynecological cancer referrals to University College London Hospital in 2012. Clinical and demographic details were collected from the Clinical Data Repository. Statistics were calculated with MeasuringU software. Among 335 women referred to the RAC, 14 (4.2\%) had cancer. Most women had benign pathology (80.6\%). This same year, 13 cancers were diagnosed elsewhere, including in the emergency department. A total of 172 referrals did not fulfill the guidelines. The apparent positive predictive value (PPV) for the referrals was 0.042. If appropriately followed, the PPV for guidelines would be 0.091 . The apparent PPV of the current referral system for urgent gynecological cancers is low. Greater discretion in primary care and proper use of referral guidelines are required. Avoiding inappropriate referrals can double the PPV, leading to cost savings for the National Health Service. Keywords: gynecology, general practice, 2-week wait, genital neoplasms, gynecological cancers

\section{Introduction}

In the National Health Service (NHS) Cancer Plan, the UK government set out a proposal to reduce delays in the diagnosis and treatment of patients with cancer. ${ }^{1} \mathrm{~A}$ component of this was to ensure that all patients referred by their GP with suspected cancer are seen by a hospital specialist within 2 weeks of the date of the letter. In response to this, the North London Gynaecological Cancer Network (NLCN) produced guidelines and a proforma for GPs to refer patients with suspected cancer to a rapidaccess clinic (RAC), to be seen by a consultant gynecological oncologist within 2 weeks of a faxed referral. ${ }^{2}$ The proforma criteria are intended for use by GPs in the primary care setting, where there is no access to colposcopy, ultrasound, or endometrial biopsy. ${ }^{3}$

Evidence suggests that only a small proportion of patients referred to the RAC have an end diagnosis of cancer. ${ }^{4-6}$ The aim of this study was to evaluate the appropriateness of GP referrals to the RAC at University College London Hospital (UCLH) and also to determine where gynecological cancers at UCLH were diagnosed, if not in the RAC.

\section{Materials and methods}

This was a retrospective review of data on referrals from primary care to the gynecology RAC at UCLH. UCLH acts as a unit hospital for the local population of 1.65 million 
in Camden and Islington and as a tertiary referral center from hospitals elsewhere. Eligible patients who attended the RAC from 1 January to 31 December 2012 were included in the study. Referrals are not triaged if they are referred to the rapid-access clinic; they are automatically given an appointment to attend within 2 weeks.

Clinical details were collected from the UCLH Clinical Data Repository (CDR). Referral indications, patient demographics (age and ethnicity), risk factors, symptoms, clinical findings, further investigations, and overall outcomes were recorded by using Microsoft Excel. Based on the referral reason indicated on the referral proforma (Table 1), the data were separated into suspected cancer types. Those without a proforma were classified based on the symptoms that they were referred with. Patients who did not attend their RAC appointment and patients who were referred for assessment of a pre-existing cancer were excluded from this study.

Final diagnosis of cervical, vulval, ovarian, or endometrial cancer was made following lesion biopsy analysis performed by histopathologists. Data on gynecological cancers that were diagnosed in the same year from other sources were obtained from the UCLH CDR. Information was reviewed to prevent data oversight.

Statistical analysis using the chi-squared test was undertaken in Microsoft Excel. The positive predictive value (PPV) was calculated by using the number of true positives as a proportion of total positive calls. True positives were when the outcome was cancer. For the apparent PPV, the total number of positive calls was the total number of rapid-access referrals. For the true PPV, the total number of positive calls was the number of rapid-access referrals that met the referral criteria set out in the NLCN proforma. Confidence intervals were calculated by using the Measuring U online calculator. ${ }^{7}$

Table I Referral criteria for the gynecology RAC

\begin{tabular}{ll}
\hline Referral information & Category of cancer \\
\hline $\begin{array}{l}\text { Lesion suspicious of cancer on cervix or vagina } \\
\text { on speculum examination or smear suggesting } \\
\text { cancer }\end{array}$ & Cervical \\
$\begin{array}{l}\text { Lesions suspicious of cancer on clinical } \\
\text { examination of vulva }\end{array}$ & Vulval \\
$\begin{array}{l}\text { Suspicious pelvic mass on pelvic ultrasound } \\
\text { (not fibroids/urological/gastrointestinal) }\end{array}$ & Ovarian \\
$>$ I or a single heavy episode of postmenopausal & Endometrial \\
$\begin{array}{l}\text { bleeding (PMB) in women }>55 \text { years not on HRT } \\
\text { HRT: unexpected or prolonged bleeding }\end{array}$ & Endometrial \\
persisting for $>6$ weeks after stopping HRT & \\
\hline
\end{tabular}

Notes: Referral criteria for the gynaecology RAC, modified from the NLCN Suspected Gynaecological Cancer Referral Form. ${ }^{2}$

Abbreviations: HRT, hormone replacement therapy; RAC, rapid-access clinic; NLCN, North London Gynaecological Cancer Network.
As this was a retrospective audit, ethical approval was not needed, and patient consent was not required to review medical records.

\section{Results}

Of the 368 patients referred to the RAC, 335 were eligible for inclusion in this study. Of these, 185 women were referred with suspected endometrial cancer, 84 for suspected ovarian cancer, 48 for suspected cervical cancer, and 18 for suspected vulval cancer. The majority of women were Caucasian (56.7\%), while $2.1 \%$ were African, 9.6\% were Asian, and $11.0 \%$ were other ethnicities. Fourteen gynecological cancers were diagnosed through the RAC referral system. The apparent PPV for the entire cohort was 0.044 (confidence interval [CI] 0.023-0.069). A total of 172 referrals did not meet the strict referral criteria and were inappropriate. If strict criteria were adhered to, the true PPV would be 0.091 (CI 0.048-0.140).

\section{Endometrial cancer}

Of the 185 women referred, the median age of women in this category was 56 (interquartile range [IQR] 13) years. The most common referring symptom was postmenopausal bleeding (PMB) in 144 (77.8\%), followed by pelvic pain in 11 (5.9\%) and intermenstrual bleeding in 8 (4.3\%). All women had an ultrasound scan, of which 71 (38.4\%) had abnormalities suspicious of endometrial cancer. Sixty seven (36.2\%) of the total had biopsies, of which 7 (10.4\%) had endometrial cancer confirmed. Overall, 7 (3.8\%) of the women referred had endometrial cancer. The other diagnoses are illustrated in Figure 1. Of the patients referred with pelvic pain, $93.8 \%$ had a benign outcome. The apparent PPV is 0.042 (CI 0.015-0.076). A total of 143 (77.3\%) were referred according to the guidelines of at least one episode of PMB in >55 years old and not on hormone replacement therapy (HRT). Therefore, the true PPV is 0.055 (CI 0.020-0.098).

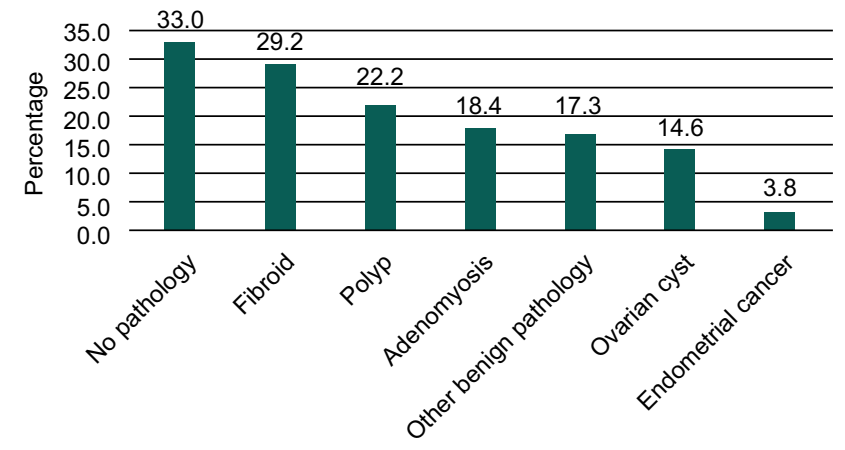

Figure I Clinical outcomes for women referred with suspected endometrial cancer. 


\section{Ovarian cancer}

Of the 84 women referred, the median age was 50 (IQR $20.75)$ years. The most common reasons for referral were pain in 34 women $(40.5 \%)$, a mass seen on ultrasound in 16 (19.0\%), bloating in $15(17.9 \%)$, an abdominal mass in 9 $(10.7 \%)$, change in bowel habit in $7(8.3 \%)$, and weight loss in $5(6.0 \%)$. The gynecologist found an abdominal mass in 14 patients (16.7\%); 78 women $(92.9 \%)$ were referred for further investigation, of which ultrasound was the most common. Overall, 4 (4.8\%) were found to have ovarian cancer. The symptoms of pain and bloating had a benign cause in $97.0 \%$ and $86.7 \%$ of cases, respectively. Other diagnoses are illustrated in Figure 2. The apparent PPV is 0.058 (CI 0.013-0.118). The referral guidelines state a suspicious pelvic mass on pelvic ultrasound scan, which was seen in 16 (19.0\%). Therefore, the true PPV is 0.278 (CI 0.072-0.524).

\section{Cervical cancer}

Of the 48 women referred, the median age was 45 (IQR 15.25 ) years; $22.9 \%$ had had a smear test within the recommended interval. Of those referred with a smear, $25(89.3 \%)$

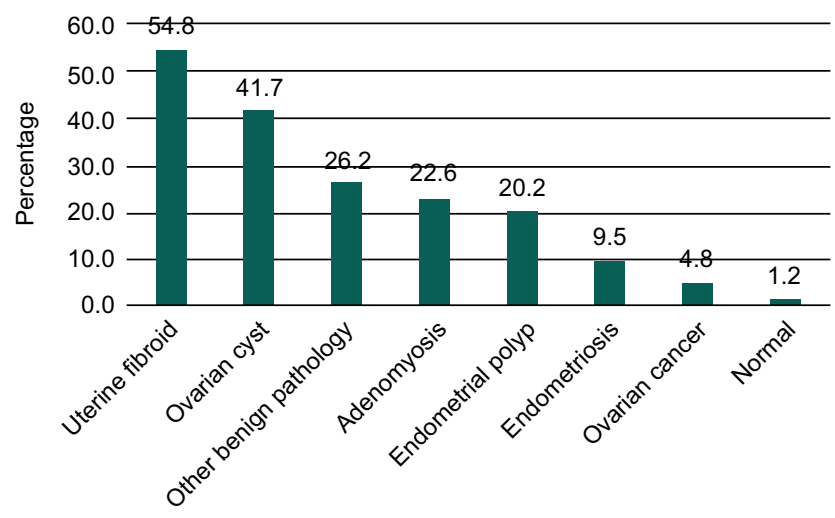

Figure 2 Clinical outcomes for women referred with suspected ovarian cancer. had a normal result, 2 (7.1\%) had mild dyskaryosis, and 1 (3.6\%) had severe dyskaryosis. The most common reasons for referral were cervical lesion/lump in 26 (54.2\%), intermenstrual bleeding in 20 (41.7\%), and postcoital bleeding in $6(12.5 \%)$. Following examination by the gynecologist, $5(10.4 \%)$ were suspected to have cervical cancer. In total, 37 (77.1\%) were investigated further, with ultrasound scan being the preferred modality in $20(41.7 \%)$, with $11(22.9 \%)$ having a biopsy. None of the women referred had cervical cancer. Two women were diagnosed with other cancers: 1 endometrial cancer and $1 \mathrm{~B}$-cell lymphoma. Other clinical outcomes are also illustrated in Figure 3. A total of 2 out of the 48 cervical cancer referrals (4.2\%) were deemed to be appropriate. The true and apparent PPV are both 0 .

\section{Vulval cancer}

Of the 18 women referred, the median age was 63 (IQR 18.75). The most common symptoms included a lump in 11 women (61.1\%), pain in $10(55.6 \%)$, and pruritis in 7 (38.9). In total, $4(22.2 \%)$ of the cases were deemed to be suspicious of vulval cancer by the gynecologist. The majority of women, $15(83.3 \%)$, had further investigations, with $11(61.1 \%)$ going on to have a biopsy. Overall, 2 women (11.1\%) were found to have vulval cancer (Figure 4). Lichen sclerosus was the most common benign outcome; $81.8 \%$ of lumps were benign and $80.0 \%$ of patients with pain had benign pathology. The apparent PPV is 0.150 (CI 0.014-0.347). The specialist found a vulval lesion suspicious of cancer in 4 ; these referrals were deemed appropriate. The true PPV is 0.500 (CI 0.068-0.932).

In total, 14 patients were diagnosed with gynecological cancer through the gynecology RAC. In the same year, 13 patients were diagnosed with gynecological cancer from other sources (Figure 5). There is no significant difference between endometrial/other types of cancer diagnosed in RAC

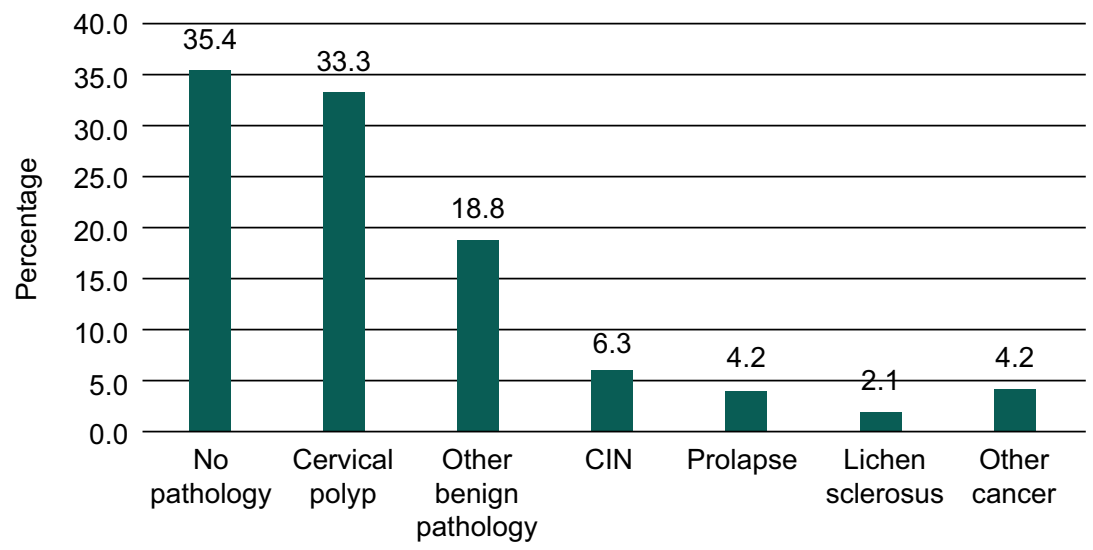

Figure 3 Clinical outcomes for women referred with suspected cervical cancer.

Abbreviation: $\mathrm{CIN}$, cervical intra-epithelial neoplasia. 


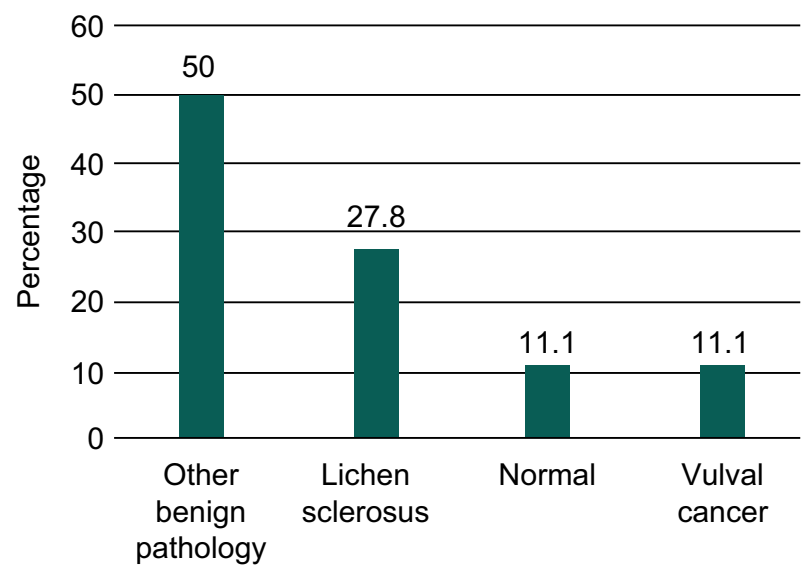

Figure 4 Clinical outcomes for women referred with suspected vulval cancer.

and from other sources ( $P=0.25$ Fisher's test). The majority of non-rapid-access referral diagnoses were made in routine gynecology clinics $(5 ; 38.5 \%)$. Other diagnoses were made in the emergency department $(4 ; 30.8 \%)$, in the cervical screening program $(1 ; 7.7 \%)$, via geriatrics clinic $(1 ; 7.7 \%)$, or incidentally $(2 ; 15.4 \%)$. Incidental diagnoses were made during abdominal surgery and following tertiary referral for an ovarian cyst. Cervical and ovarian cancers were more likely to be diagnosed outside of the RAC, with vulval and endometrial cancers diagnoses more often in it.

\section{Discussion}

The purpose of the RAC is to detect and diagnose cancers promptly, with the ultimate aim of saving more lives. ${ }^{1}$ There is a commitment to improving cancer outcomes, and a lot of resources have been made available to support this. Despite this, the UK continues to lag behind other developed countries, and it is important to critically review the pathway designed for prompt diagnosis of cancer. This study demonstrates a low yield of cancer diagnoses via the 2-week wait referral system.

The majority of women seen in the RAC at UCLH had benign pathology. Symptoms such as pain and bloating were referred most often with benign outcomes. At the time of data collection, there was no guidance regarding the expected proportion of malignant versus benign disease; but it is of concern that obvious benign pathology such as ectropion and fibroids are being referred as suspected cancer. The 2015 update of National Institute for Health and Care Excellence cancer recognition and referral guidelines suggests a PPV of at least $3 \%$ for all cancers. ${ }^{8}$ Therefore, the PPV of 0 for cervical cancer is particularly poor. This may be an indication that more focused training is required to aid better recognition of benign pathology in the community, in order to prevent unnecessary referrals to the RAC. Whilst these women may need to be referred to a gynecological clinic, a 2-week wait referral is not appropriate.

This low predictive value has also been seen in rapidaccess clinics for other types of cancer. ${ }^{9}$ Results from this study and evidence from literature suggest that a high proportion of gynecological cancers are being diagnosed outside of the RAC, and many in routine outpatient clinics and the emergency department ${ }^{5,10}$ (Figure 5). A bench marking paper comparing the 1- and 5-year survival in 4 key cancers in Australia, Canada, Denmark, Norway, Sweden, and the UK showed the UK lagged behind in 1- and 5-year survival rates for ovarian cancer. ${ }^{11}$ One-year survival rates reflect diagnosis with advanced disease. Higher presentation rates in emergency department are considered to be a key factor in this.

It has been shown that the majority of patients with cancer who present to their GP are referred to a hospital specialist within 1 or 2 visits. However, a quarter had 3 or

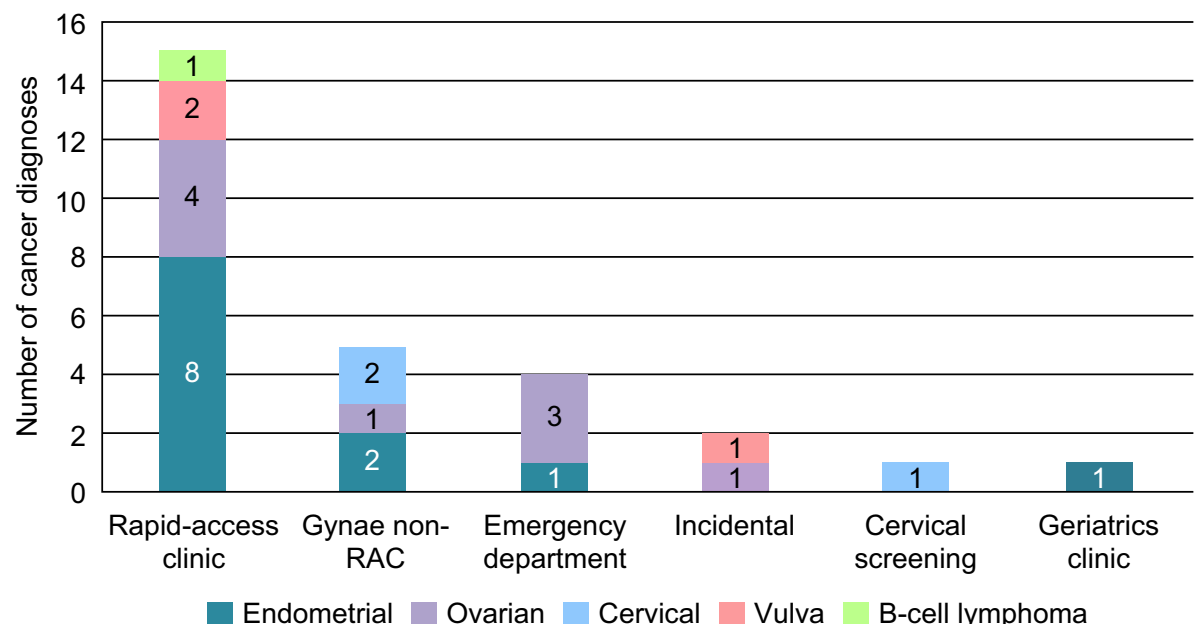

Figure 5 Location of all gynecological cancer diagnosis in 2012 at UCLH.

Abbreviations: RAC, rapid-access clinic; UCLH, University College London Hospital. 
more consultations before being referred. This reflects those cancers that have more nonspecific symptoms, including ovarian cancer. ${ }^{12}$

The introduction of the 2-week wait referral system has had a significant impact on the workload of gynecologists. It has been shown that this has impacted time and resources available to other patients, increasing waiting times for routine outpatient appointments. ${ }^{10}$ This effect has also been seen in urgent GP referrals for other types of cancer. ${ }^{13,14}$ Since many cancers are being detected outside of the 2-week wait system, this could lead to delay in diagnosis of these cancers. Furthermore, patients with benign pathology can experience unnecessary anxiety through being referred to the RAC. However, it is also important to consider the patient value of early specialist assessment in reducing anxiety and the risks of delaying a diagnosis of cancer. ${ }^{15}$

This study addresses important clinical questions surrounding the appropriateness of referrals and efficiency of the rapid-access cancer pathway. The strengths include the systematic inclusion of all cases referred through the RAC pathway and seen in a cancer center over the period of a year. The limitation of this study is that the data were analyzed retrospectively, leading to some degree to subjective interpretation of the referral intent.

\section{Conclusion}

The apparent predictive value of urgent gynecological referral guidelines is low. An ideal health service would ensure that all patients are seen promptly with no artificial distinction between probable malignant and benign disease. As this is not currently achievable, it is important that GPs familiarize themselves with the range of benign appearances to minimize both inappropriate referrals and patient anxiety and improve efficiency of the referral pathways. The data of this study suggest that the PPV of the referral pathway can be doubled just by "appropriate" referral of patients. This small implementable step itself can lead to significant cost savings and increase the efficiency of the cancer referral pathway.

\section{Disclosure}

The authors report no conflicts of interest in this work.

\section{References}

1. Department-of-Health. The NHS Cancer Plan: a plan for investment, a plan for reform; 2000. Available from: http://webarchive.nationalarchives.gov.uk/+/www.dh.gov.uk/en/Publicationsandstatistics/Publications/PublicationsPolicyandGuidance/DH_4009609. Accessed May 9, 2017.

2. How to Make a Referral; 2014. Available from: http://www.uclhwomenscancer.com/how-to-make-a-referral.asp\#. Accessed November, 2014.

3. Harrison M, Adebiyi A, Lyddiard J, Bridgewater J. North London Cancer Research Network Annual Report 2012-2013; 2013. Available from: http://www.londoncancer.org/media/62730/north-london-cancerresearch-network-annual-report-2012-2013-final.pdf. Accessed May 9, 2017.

4. Jones R, Rubin G, Hungin P. Is the two week rule for cancer referrals working? BMJ. 2001;322(7302):1555-1556.

5. Lewis R, Collins R, Flynn A, et al. A systematic review of cancer waiting time audits. Qual Safe Health Care. 2005;14(1):62-66.

6. Burbos N, Musonda P, Rufford B. Diagnostic performance of urgent referrals for suspected gynaecological malignancies. Arch Gynecol Obstet. 2011;284(6):1495-1500.

7. Sauro J. Confidence interval calculator for a completion rate; 2005 . Available from: http://www.measuringu.com/wald.htm\#marg. Accessed July 13,2015

8. NICE. Suspected cancer: recognition and referral (NG12); 2015. Available from: https://www.nice.org.uk/guidance/ng12. Accessed May 9, 2017.

9. Allgar VL, Neal RD, Ali N, et al. Urgent GP referrals for suspected lung, colorectal, prostate and ovarian cancer. Br J Gen Pract. 2006;56(526) 355-362.

10. Morrison J. 'Two week wait' standards for suspected gynaecological malignancy. On target, but missing the point? $J$ Br Menopause Soc. 2003;9(4):170-172.

11. Coleman MP, Forman D, Bryant H, et al. Cancer survival in Australia, Canada, Denmark, Norway, Sweden, and the UK, 1995-2007 (the International Cancer Benchmarking Partnership): an analysis of populationbased cancer registry data. Lancet. 2011;377(9760):127-138.

12. Mayor S. A quarter of patients with cancer see their GP several times before being referred. BMJ. 2011;343:d7601.

13. Hanna SJ, Muneer A, Khalil KH. The 2-week wait for suspected cancer: time for a rethink? Int J Clin Pract. 2005;59(11):1334-1339.

14. Potter S, Govindarajulu S, Shere M, et al. Referral patterns, cancer diagnoses, and waiting times after introduction of two week wait rule for breast cancer: prospective cohort study. BMJ. 2007;335(7614): 288.

15. Cornford CS, Harley J, Oswald N. The '2-week rule' for suspected breast carcinoma: a qualitative study of the views of patients and professionals Br J Gen Pract. 2004;54(505):584-588.

\section{Clinical Audit}

\section{Publish your work in this journal}

Clinical Audit is an international, peer-reviewed, open access journal focusing on the processes and outcomes of clinical audit in any area of healthcare. All aspects of patient care are addressed within the journal and practitioners from all disciplines are invited to submit their work. Areas covered include: Publication of audits; How an audit has changed practice;
Practical tips on how to do audits and to avoid pitfalls; How audits have changed patient care; Calls and justifications for new audits. The manuscript management system is completely online and includes a very quick and fair peer-review system, which is all easy to use. Visit http://www.dovepress. com/testimonials.php to read real quotes from published authors. 